\title{
Williams-Otto Plant Control Based on Production Planning Associated to Coordinated Decentralized Optimization and Plantwide Control Techniques
}

\author{
Anamaria de Oliveira Cardoso ${ }^{1 *}$ and Wu Hong Kwong ${ }^{2}$ \\ 1. Science and Technology Institute, Federal University of Jequitinhonha and Mucuri Valleys, Diamantina, 39100-000, Brazil \\ 2. Department of Chemical Engineering, Federal University of São Carlos, São Carlos, 13565-905, Brazil
}

\begin{abstract}
Manufacturing plants are increasingly complex and integrated, requiring control systems able to identify the interactions between the various operating units. Production planning and control design of a process are tools that, if combined, bring many economic benefits to the processes since they aim to identify and maintain optimal decision operations to a system. This work uses such integration between production planning and plantwide control to propose a control system for the Williams-Otto plant from the definition of the operating optimal point for coordinated decentralized optimization, in which the original optimization problem decomposition into smaller coordinated problems ensure that the found local optimum also meets the requirements of the global system. The results for decentralized optimization are satisfactory and very similar to the global optimum problem and to the control system response proposed based on the optimal obtained. It is effective taking smooth actions, working with (economic) optimal set points (economically) of operation. The unification of production planning techniques and plantwide control techniques is an effective tool for the control system design for entire plants.
\end{abstract}

Key words: Coordination, decentralized optimization, plantwide control, large-scale systems.

\section{Introduction}

An industrial plant has dozens of processing units, increasingly integrated and complex, in which local control decisions cover the entire plant, resulting in several control loops alternatives to the many possibilities of measured, controlled and manipulated variables [1, 2].

Interactions between units via recycle streams or energy integration systems have been the focus of several studies over the past decades. The isolated behavior combination of the units of a process does not represent the dynamic behavior of an entire plant. Such interactions can lead to slow responses or to a high sensitivity of the stationary state and even instability [3]. Therefore, it is necessary to develop

\footnotetext{
*Corresponding author: Anamaria de Oliveira Cardoso, master, research fields: modeling, simulation, control and optimization of chemical process.
}

efficient control strategies for entire plants that consider interactions between its processing units [3-5].

The control of an entire plant aims to determine a complex industrial plant control structure. Setting manipulated variables and control objective appropriate measures, considering the interactions of the plant and its complex dynamics and determining the configuration and type of the appropriate control to such system are among the stages of this kind of project [6-8].

Synthesizing control systems for entire plants has become an increasing challenge and several studies have made significant effort to develop successful strategies for that.

Stephanopoulos and Ng [8], Larsson and Skogestad [9] present a meaningful review of the research developed in this area, highlighting the first work done by Buckley [10] on dynamic process control. The author proposes the decomposition of the problem based on 
different timescales, thus defining two stages in the project control process: material balance control (fast dynamics) and product quality control (slow dynamics). This work disintegrates the control problem decomposition into smaller subproblems as a strategy for plantwide control project.

W. L. Luyben, Tyréus, and M. L. Luyben [11] define several heuristics for a synthesis of entire plant control systems and some studies are based on optimization techniques, such as Narraway and Perkins [12] Skogestad, Araujo [7, 8] and Degliuomini, Zumoffen and Basualdo [13].

Designing a safe and profitable chemical plant requires efficient and well-defined control strategies to ensure a good process performance. For that, it is important to consider operational and economic aspects to establish operating conditions that satisfy both criteria.

Some structures were presented over the past years, combining control and process optimization in order to improve production aspects, such as quality and energy efficiency, along with compliance with safety and environment laws [1].

Developing methodologies for the definition of operating conditions where marketing and safety decisions are integrated with the unit process operations from their optimization is still an unexplored problem for the entire plant control system, and it can be an effective alternative to do this [14, 15].

In this context, this paper presents the integration of planning concepts (scheduling) and plantwide controlling to define operation optimal conditions (economically) from Williams-Otto coordinate decentralized optimization and plantwide control system project $[16,17]$.

\section{Decentralized Coordinated Optimization to Large Scale Systems}

\subsection{Decentralized Optimization}

The control system of a process is a decision-making hierarchical system and may be classified into levels with different operation times, as shown in Fig. 1.

While the predictive control operates in minutes, the upper levels, directly related to the optimization problem solving in order to determine the set points of the control lower layers, have long operating hours, days or even weeks [9, 11-17].

To ensure interaction and applicability of decision-making actions between levels, it is essential to adopt strategies so that the results are accurate and meet the restrictions. Therefore, several studies propose effective techniques for solving optimization problems in large scale, with emphasis on non-linear systems [19-21].

The decomposition of large-scale systems into smaller problems is presented as methodology in addressing such problems. This strategy reduces the complexity of the problem and the computational effort required, finding solutions around $1 \%-3 \%$ of the global optimum [21], satisfying the economic and operational criteria [22].

Previous studies introduce several decentralized optimization applications for chemical processes. Grossmann and Harjunkoski use the decomposition for the planning of a steel plant [21]. Shah and

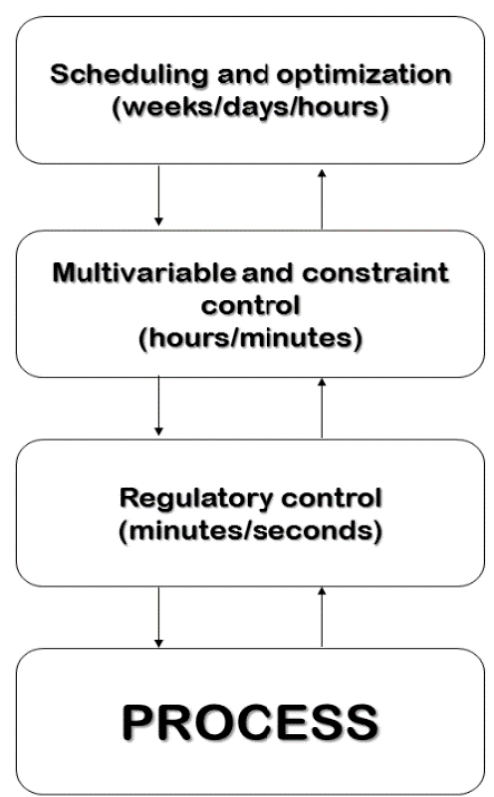

Fig. 1 Hierarchical control system to chemical process (adapted by Skogestad [6]; Baldea and Harjunkoski [15]). 
colleagues [23] use the centralized optimization with decentralized optimization for the planning of a refinery achieving the same result applying both methods. Gnoni and colleagues use a hybrid approach, combining mixed-integer linear programming model and simulation for a multi-site manufacturing process planning of the automotive industry, obtaining promising results with decentralized optimization [24].

For chemical processes, decomposition is determined, in most cases, as an empirical and natural way, according to the structure of the process. In this work it was used the studied decomposition process based on the separability of the constraints and the objective function that involves the optimization problem. Section 3 presents the decomposition used in this work.

Consider the global optimization problem Eq. (1) to determine controlled variables optimal set points for the process of interest:

$$
\min _{u} \Phi(x, u, d)
$$

subject to

$$
\begin{aligned}
& f(x, u, d)=0 \\
& g(x, u, d) \leq 0
\end{aligned}
$$

where, $x$ is the system state vector; $u$ is the input vector and $d$ are disturbances to which the system is subject to. Consider that the objective function $\Phi$ can be decomposed additively as shown in Eq. (2):

$$
\Phi=\sum_{\mathrm{i}=1}^{\mathrm{N}} \Phi_{\mathrm{i}}
$$

and $\Phi_{i}$ is the portion of the objective function corresponding to the subsystem $i$.

However, it is essential that the optimal location solution also meet the demands of the global system. For that, it is necessary to coordinate the resolution of such sub-problems considering that chemical plants are complex systems with a strong interaction between the units that compose them.

\subsection{Coordination}

The coordination of local optimization problems assures that the results of decentralized optimization also achieve the common goal and not just the local demands of each subsystem, preventing them to conflict with each other [25].

Fig. 2 presents the concepts of multilayer decomposition control tasks of a chemical plant.

Findeisen and co-workers [26] have two coordination categories based on the system model without any return of the real process: the first method known as Direct Method and the second method, the IBM (Interactions Balancing Method).

The IBM attempts to minimize the differences between the connection variables (inputs and outputs) of the subsystems. For that, the coordinator ought to maintain the interconnection equations of the subsystems:

$$
u_{i}=\sum_{i} Q y_{i}
$$

Lagrangian function formed by the global performance index and the total interconnections is the basis of the method. Thus, $N$ local optimization problems, Eqs. (4) and (5), compose the decentralized optimization:

$$
\min _{u} \varphi_{i}\left(x_{i}, u_{i}, d_{i}, \lambda\right)
$$

subject to

$$
\begin{aligned}
& f_{i}\left(x_{i}, u_{i}, d_{i}\right)=0 \\
& g_{i}\left(x_{i}, u_{i}, d_{i}\right) \leq 0
\end{aligned}
$$

Define:

$$
\varphi_{i}=\Phi_{i}-\lambda_{i}^{T} u_{i}+\sum_{j}^{N} \lambda_{j}^{T} Q_{i j} y_{i}
$$

From such method, the multipliers $\lambda_{i}$ are initially estimated in the coordination from the optimization of

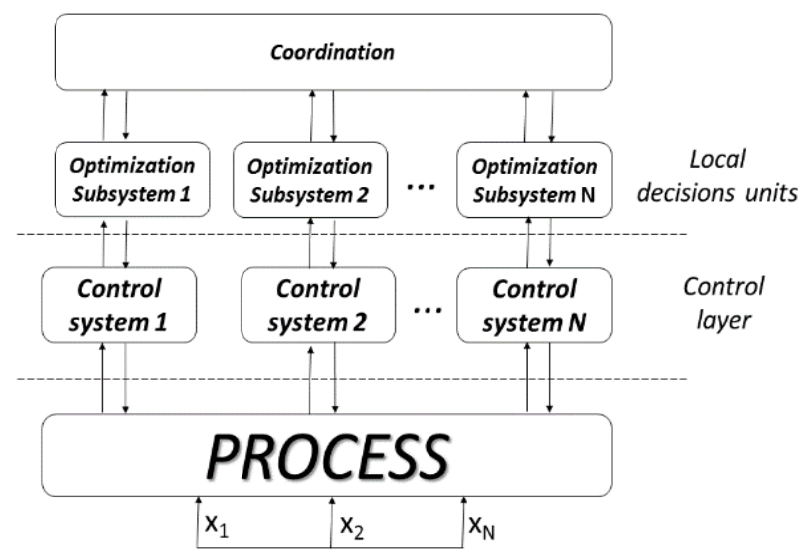

Fig. 2 Coordinated decentralized optimization of the control tasks of a process. 
the subsystems; an initial estimate of such variables is needed for the coordination determine them. This is a drawback, because there is no reference on which values $\lambda_{i}$ can assume. However, several studies show that the method is more accurate than others coordination methods and presents satisfactory results in large-scale system optimization [14, 26].

The Direct Method is based on a price strategy, in which the coordinator specifies the outputs $y_{i}$ for the subsystems and seeks to solve the optimization problem.

$$
\min _{y_{s p}} \Phi(x, u, d)=\sum_{i=1}^{N} \Phi_{i}
$$

The local optimization of each subsystem is defined

$$
\min _{x} \Phi_{i}\left(x_{i}, u_{i}, d_{i}\right)
$$

subject to

$$
\begin{gathered}
u_{i}=\sum_{j} Q_{i j} y_{i, s p} \\
g_{i}\left(x_{i}, u_{i}, d_{i}\right) \leq 0
\end{gathered}
$$

Several studies use the hierarchical control with the coordination to obtain satisfactory results in process control; however, only a few associate the decentralized optimization with the coordination to determine the stationary state point for the controller.

Bakallis and Ellis [27] applied four types of coordination strategies to a vaporizer on a pilot scale, decomposed into two interconnected subsystems, obtaining satisfactory results with the implementation of the Direct and the IBM. Flórez and co-workers [28] designed a control structure for a hydropower valley using the model-based predictive controller coordinated from the IBM. The local optimization stage to find the optimal point of operation was not used and the proposal led to a reduced computational cost.

In order to set optimization framework for systems of interest, it is important to note that in the Direct Method, the initial estimates for the coordinator are connection variables. Centralized optimization requires one to know all the initial estimates to variables involved in optimization problems that, in many times, are not measurable.
Therefore, the method is more easily implementable in practice, variables of connections are measured on the instant at which the optimization is needed and adopted like initial estimates to the method.

\section{Results and Discussions}

\subsection{Coordinated Decentralized Optimization Applied to Williiams-Otto Plant}

Williams-Otto plant has been the focus of several studies due to the complexity presented by the optimization problem that it involves. Fig. 3 shows the process of Williams-Otto plant used in this study.

By means of simplification, it is considered that the reactor is a CSTR (Continuous Stirred Tank Reactor) and that the cooling caused by its jacket is sufficient, thereby excluding the heat exchanger shown in the original work [15]. In the reactor, the following reactions occur:

$$
\begin{gathered}
A+B \stackrel{k_{1}}{\rightarrow} C \\
C+B \stackrel{k_{2}}{\rightarrow} P+E \\
P+C \stackrel{k_{3}}{\rightarrow} G
\end{gathered}
$$

The constant of the reaction is $k_{i}=A_{i} e^{-B_{i} / T_{r}}$. It is known that

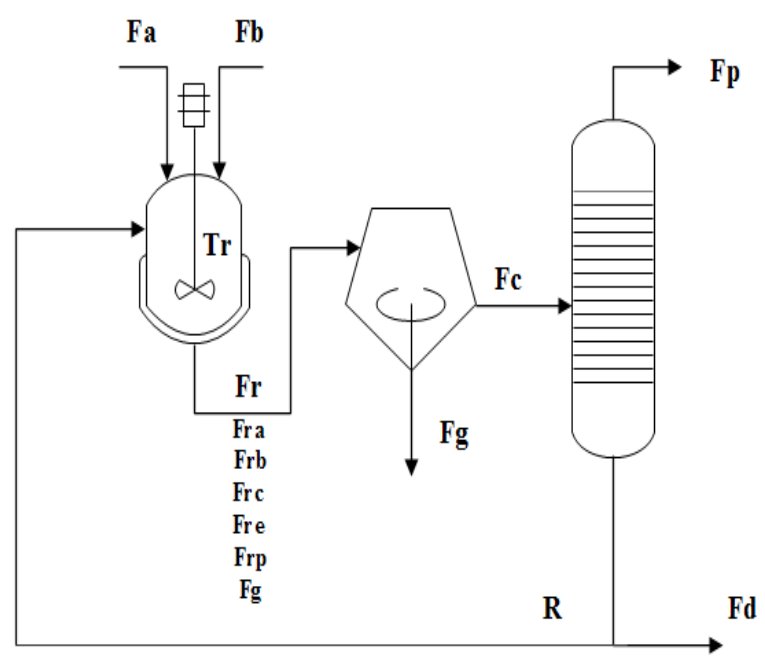

Fig. 3 Williams-Otto plant. 


$$
\begin{gathered}
A_{1}=5.9755 \times 10^{9} / h, w t . \text { fraction; } \\
B_{1}=12000^{\circ} \mathrm{R}(\text { baseado em } A \text { ou } B), \\
A_{2}=2.5962 \times 10^{12} / h, w t . \text { fraction; } \\
B_{2}=15000^{\circ} \mathrm{R}(\text { baseado em } B), \\
A_{3}=9.6283 \times 10^{15} / h, w t . \text { fraction; } \\
\left.B_{3}=20000^{\circ} \mathrm{R} \text { (baseado em } C\right)
\end{gathered}
$$

For operational and safety criteria, $580^{\circ} R \leq T_{R} \leq$ $680^{\circ} R$. The system reaction rates are set:

$$
\begin{aligned}
& r_{1}=k_{1} \frac{F_{R A} F_{R B}}{F_{R}^{2}} \\
& r_{2}=k_{2} \frac{F_{R B} F_{R C}}{F_{R}^{2}} \\
& r_{3}=k_{3} \frac{F_{R C} F_{R P}}{F_{R}^{2}}
\end{aligned}
$$

$\mathrm{F}_{\mathrm{R} i}$ is an output reactor flow rate of the $i$ compound. $P$ is a product of interest and $G$ is a byproduct discarded after treatment that results in additional cost to the process. The availability of raw materials is limited: $\quad 12400 \mathrm{lb} / \mathrm{h} \leq F_{A} \leq 16600 \mathrm{lb} / \mathrm{h}$ and $F_{B} \leq 56000 \mathrm{lb} / \mathrm{h}$.

The optimization problem is to increase the current rate of return on investment of the process, represented by the objective function, considering that the price of utilities is related to the column inlet flow rate and to the recycle flow rate [17]:

$$
I(\%)=100 \frac{[M-S A R E-U-F C P]}{P I N}
$$

Define:

$$
\mathrm{M}=8400\left(P_{P} F_{P}+P_{D} F_{D}-P_{A} F_{A}-P_{B} F_{B}-\right.
$$

$\left.P_{G} F_{G}\right)$-Gross return per hour

SARE $=(0.124)(8400)\left(P_{P} F_{P}+P_{D} F_{D}\right)-$ Sales, administration, research and engineering charges

$$
\mathrm{U}=8400\left(C_{1} R^{1,5}+C_{2} F_{C}{ }^{1,5}\right)-\text { Utility charges, }
$$

which can be tied to plant flow rates

$F C P=60 \mathrm{~V} \rho-$ Fixed charges (depreciation, labor, etc.)

PIN $=600 \mathrm{~V} \rho-$ Total investments.

$F_{i}$ e $P_{i}$ are flow rates and unit prices of each flow.

The mass balance of the components involved in the reactions represents a dynamic behavior of the system [15]:

$\frac{V_{R} \rho_{R}}{F_{R}} \frac{d F_{R A}}{d t}=F_{A}+R\left(\frac{F_{R A}}{F_{R}-F_{G}-F_{P}}\right)-F_{R A}-r_{1} V_{R} \rho_{R}$

$$
\begin{gathered}
\frac{V_{R} \rho_{R}}{F_{R}} \frac{d F_{R B}}{d t}=F_{B}+R\left(\frac{F_{R B}}{F_{R}-F_{G}-F_{P}}\right)-F_{R B}-r_{1} V_{R} \rho_{R}- \\
\frac{V_{R} \rho_{R}}{F_{R}} \frac{d F_{R C} \rho_{R}}{d t}=R\left(\frac{F_{R C}}{F_{R}-F_{G}-F_{P}}\right)-F_{R C}+2 r_{1} V_{R} \rho_{R}- \\
\frac{2 r_{2} V_{R} \rho_{R}-r_{3} V_{R} \rho_{R}}{\frac{V_{R} \rho_{R}}{F_{R}} \frac{d F_{R E}}{d t}=}=R\left(\frac{F_{R E}}{F_{R}-F_{G}-F_{P}}\right)-F_{R E}+2 r_{2} V_{R} \rho_{R} \\
\frac{V_{R} \rho_{R}}{F_{R}} \frac{d F_{R C}}{d t}=R\left(\frac{F_{R P}-F_{P}}{F_{R}-F_{G}-F_{P}}\right)-F_{R P}+r_{2} V_{R} \rho_{R}- \\
0.5 r_{3} V_{R} \rho_{R} \\
\frac{V_{R} \rho_{R}}{F_{R}} \frac{d F_{G}}{d t}=-F_{G}-1.5 r_{3} V_{R} \rho_{R}
\end{gathered}
$$

The reactor is isothermal with constant volume and considers the pseudo-stationary system; subsystem reaction dynamics is slower than separation system dynamics thus, considering the last one in stationary state. The values for the model parameters are available in Williams and Otto research [16].

In addition to the six mass balances for species carried out in the reactor, the optimization problem also includes the definition of $F_{R}$ and the global mass balance system as constraints. The efficiency of separation column is:

$$
F_{P}=F_{R P}-0.1 F_{R E}
$$

Two subsystems form Williams-Otto plant, determined in accordance with Fig. 4. The optimal steady state of the plant is determined by decentralized optimization with the Direct Method and the IBM to coordinate the solutions of optimization of local problems.

Subsystem 1 has 10 restrictions, including the overall mass balance, mass balance for the subsystem and the decanter balance, besides the definition of $F_{R}$. Subsystem 2 contains only the overall mass balance of the subsystem as a restriction. 


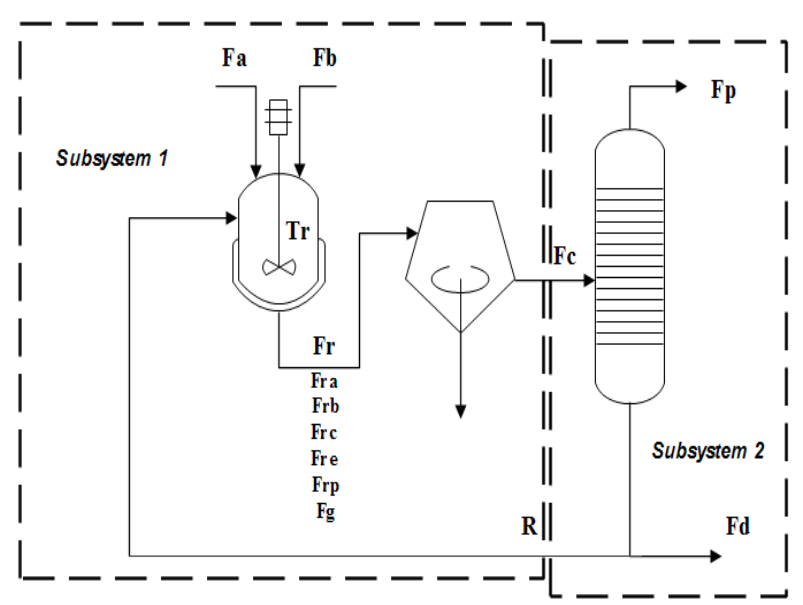

Fig. 4 Williams-Otto plant decomposed into two subsystems.

It was used the value of fixed $F_{P}$ at the optimal value equal to $4763 \mathrm{lb} / \mathrm{h}$ and SP3 point as an initial estimate presented in [16]. To solve the problem, it is adopted $P_{P}=\$ 0.3(l b / h)^{-1}, P_{A}=\$ 0.02(l b / h)^{-1}, P_{B}=$ $\$ 0.03(l b / h)^{-1} \quad, \quad P_{G}=\$ 0.01(l b / h)^{-1}$ e $P_{D}=$ $\$ 0.0068(l b / h)^{-1}$

Table 1 shows results for centralized and decentralized optimization coordinated with the Direct Method and the IBM. It is noteworthy that both used the SQP algorithm to solve the problem and initial estimate to $\lambda_{0}=\left[\begin{array}{ll}8.10^{-4} & 8.10^{-6}\end{array}\right]^{T}$.

It is noticed that the decentralized optimization promotes an increase of $0.03 \%$ in the yield of the plant. Results of decentralized optimization are similar to the optimal point defined by the centralized problem, with small adaptations in these values to ensure that restrictions of subsystems are complied.

The decentralized optimization promotes a slightly higher yield of the plant when coordination of optimization uses the Direct Method.

The IBM promotes a $0.9 \%$ increase in yield. Results of decentralized optimization are similar to the optimal point defined by the centralized problem, with small adaptations in these values to ensure that restrictions of subsystems are complied.

In order to simulate changes that have occurred in the market in practice, thus affecting the optimal operating point, the authors have changed the treatment cost value of the waste generated by the process $F_{G}$. In the new optimization scenario, $P_{G}=\$ 0.03(l b / h)^{-1}$. The steady states determined by optimization methods (Table 1 ) are the initial estimates to a new problem and $\lambda_{0}=\left[\begin{array}{ll}7.10^{-4} & 8.10^{-6}\end{array}\right]^{T}$, the optimal steady states to this scenario are in Table 2.

In this case, decentralized optimization promotes an increase of $0.11 \%$ and $2.72 \%$ in the yield of the plant to the Direct Method and the IBM, respectively. Over again, decentralized coordinated optimization achieved similar results when compared with centralized optimization, however, reaching an even higher yield than the centralized optimization.

Table 1 Steady states defined by optimization for Williams-Otto plant to $P_{G}=\$ 0.01(l b / h)^{-1}$.

\begin{tabular}{llll}
\hline & Centralized & Direct Method & IBM \\
\hline $\mathbf{F}_{\mathbf{A}}$ & $13,545.287$ & $13,552.529$ & $13,100.087$ \\
$\mathbf{F}_{\mathbf{B}}$ & $31,506.085$ & $31,505.836$ & $32,025.464$ \\
$\mathbf{F}_{\mathbf{R}}$ & $157,291.428$ & $157,290.365$ & $159,591.265$ \\
$\mathbf{F}_{\mathbf{R A}}$ & $18,166.939$ & $18,213.978$ & $17,815.840$ \\
$\mathbf{F}_{\mathbf{R B}}$ & $60,810.825$ & $60,827.544$ & $65,337.550$ \\
$\mathbf{F}_{\mathbf{R C}}$ & $3,331.909$ & $3,343.841$ & $3,359.858$ \\
$\mathbf{F}_{\mathbf{R E}}$ & $60,554.496$ & $60,494.216$ & $59,239.852$ \\
$\mathbf{F}_{\mathbf{R P}}$ & $10,818.450$ & $10,812.422$ & $10,686.985$ \\
$\mathbf{F}_{\mathbf{G}}$ & $3,608.809$ & $3,598.365$ & $3,151.179$ \\
$\mathbf{F}_{\mathbf{D}}$ & $36,679.563$ & $36,696.999$ & $36,697.002$ \\
$\mathbf{T}_{\mathbf{R}}$ & 655.893 & 655.765 & 653.691 \\
$\mathbf{R}$ & - & $112,232.000$ & $115,394.058$ \\
$\mathbf{F C}$ & - & $153,691.999$ & $153,692.001$ \\
$\mathbf{I}(\mathbf{\%})$ & $\mathbf{8 8 . 3 3 3}$ & $\mathbf{8 8 . 3 6 6}$ & $\mathbf{8 9 . 1 4 1}$ \\
\hline
\end{tabular}


Table 2 Steady states defined by optimization for Williams-Otto plant to $P_{G}=\$ 0.03(l b / h)^{-1}$.

\begin{tabular}{llll}
\hline & Centralized & Direct Method & IBM \\
\hline $\mathbf{F}_{\mathbf{A}}$ & $13,550.959$ & $13,552.532$ & $13,586.004$ \\
$\mathbf{F}_{\mathbf{B}}$ & $31,503.076$ & $31,505.836$ & $32,408.643$ \\
$\mathbf{F}_{\mathbf{R}}$ & $157,297.190$ & $157,290.363$ & $161,338.511$ \\
$\mathbf{F}_{\mathbf{R A}}$ & $18,191.913$ & $18,214.003$ & $20,343.744$ \\
$\mathbf{F}_{\mathbf{R B}}$ & $60,800.771$ & $60,827.556$ & $67,229.757$ \\
$\mathbf{F}_{\mathbf{R C}}$ & $3,337.433$ & $3,343.848$ & $4,008.774$ \\
$\mathbf{F}_{\mathbf{R E}}$ & $60,543.003$ & $60,494.179$ & $56,614.468$ \\
$\mathbf{F}_{\mathbf{R P}}$ & $10,817.300$ & $10,812.418$ & $10,424.447$ \\
$\mathbf{F}_{\mathbf{G}}$ & $3,606.769$ & $3,598.359$ & $2,717.321$ \\
$\mathbf{F}_{\mathbf{D}}$ & $36,684.265$ & $36,697.005$ & $36,697.002$ \\
$\mathbf{T}_{\mathbf{R}}$ & 655.849 & 655.765 & 647.804 \\
$\mathbf{R}$ & - & $112,231.993$ & $115,343.864$ \\
$\mathbf{F C}$ & - & $153,692.005$ & $153,692.001$ \\
$\mathbf{I}(\mathbf{\%})$ & $\mathbf{5 5 . 1 7 0}$ & $\mathbf{5 5 . 2 7 1}$ & $\mathbf{5 6 . 1 9 7}$ \\
\hline
\end{tabular}

\subsection{Control System}

The selection of manipulated and controlled variables of the plant was based on the methodology proposed by W. L. Luyben, Tyréus, and M. L. Luyben [11], in order to avoid the so-called snowball effect. Fig. 5 presents a proposed control system to Williams-Otto plant.

The levels of the reactor and column are kept constant, as well as the ratio between the reactant feed flows $\mathrm{A}$ and $\mathrm{B}$.

The levels of the reactor and the column are held constant from the manipulation of $F_{R}$ and $F_{D}$, while the recycle flow is maintained in steady state value to it, determined in optimization layer.

The reactant feed flow rate was selected as manipulated variable to hold the flow of $\mathrm{P}$ produced in the reactor $\left(F_{R P}\right)$ in its great value in order to ensure that $F_{P}$ is in the stipulated amount. The flow rate of $\mathrm{B}$ is always maintained at the same ratio in relation to the flow rate of $\mathrm{A}$, which is in the steady-state point $\left(F_{A s s} / F_{B s S}\right)$, according to control systems settings to $F_{A}$ at each instant of time.

The temperature is manipulated variable in order to maintain the production of the by-product $G$ at its optimal point. Two PI controllers are designed for those control loops.

The simulated scenario indicates the variation in processing values of the flow rate $F_{G}$, directly linked to the treatment of waste generated by the process, by $P_{G}=\$ 0.01(l b / h)^{-1}$, in scenario 1 , to $P_{G}=$ $\$ 0.03(l b / h)^{-1}$, in scenario 2. The other parameters of the objective function kept the values presented in section 3.1 constant.

In every moment of change of the mentioned variables, it is necessary to activate the decentralized optimization to perform a new planning of the plant from the point in which it lies. Table 3 presents the optimal values of steady states obtained in optimization layer using decentralized coordinated optimization with the Direct Method.

It was determined three stationary states, considering that initially it is used scenario 1 as parameter

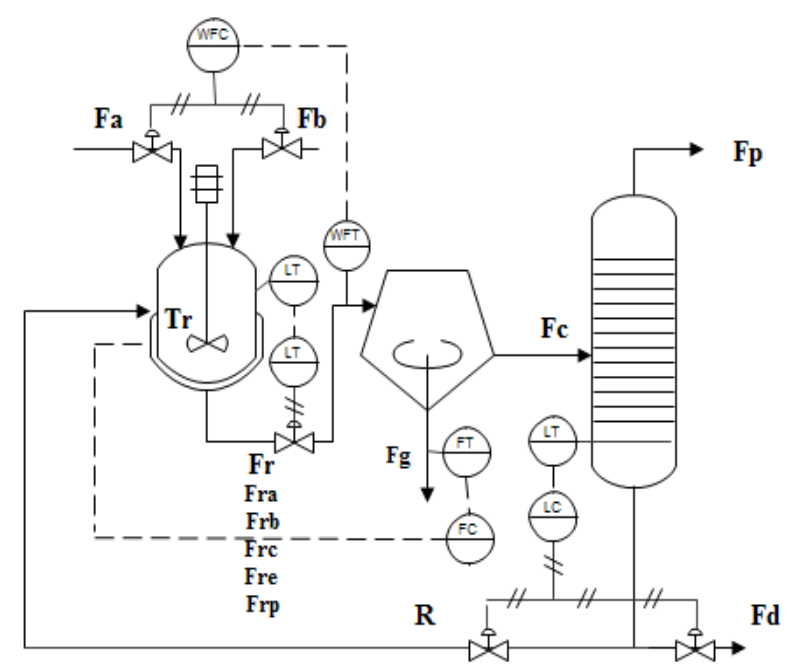

Fig. 5 Proposed control system to Williams-Otto plant. 
Table 3 Steady states defined by decentralized coordinated optimization with Direct Method for the control system of the Williams-Otto plant.

\begin{tabular}{llll}
\hline & $\boldsymbol{S S}_{\mathbf{1}}$ & $\boldsymbol{S S}_{\mathbf{2}}$ & $\boldsymbol{S S}_{\mathbf{3}}$ \\
\hline $\mathbf{F}_{\mathbf{A}}$ & $13,552.529$ & $13,552.532$ & $13,552.532$ \\
$\mathbf{F}_{\mathbf{B}}$ & $31,505.836$ & $31,505.836$ & $31,505.836$ \\
$\mathbf{F}_{\mathbf{R}}$ & $157,290.365$ & $157,290.363$ & $157,290.361$ \\
$\mathbf{F}_{\mathbf{R A}}$ & $18,213.978$ & $18,214.003$ & $18,214.010$ \\
$\mathbf{F}_{\mathbf{R B}}$ & $60,827.544$ & $60,827.556$ & $60,827.557$ \\
$\mathbf{F}_{\mathbf{R C}}$ & $3,343.841$ & $3,343.848$ & $3,343.849$ \\
$\mathbf{F}_{\mathbf{R E}}$ & $60,494.216$ & $60,494.179$ & $60,494.170$ \\
$\mathbf{F}_{\mathbf{R P}}$ & $10,812.422$ & $10,812.418$ & $10,812.417$ \\
$\mathbf{F}_{\mathbf{G}}$ & $3,598.365$ & $3,598.359$ & $3,598.360$ \\
$\mathbf{F}_{\mathbf{D}}$ & $36,696.999$ & $36,697.005$ & $36,697.008$ \\
$\mathbf{T}_{\mathbf{R}}$ & 655.765 & 655.765 & 655.765 \\
$\mathbf{R}$ & $112,232.000$ & $112,231.993$ & $112,231.990$ \\
$\mathbf{F C}$ & $153,691.999$ & $153,692.005$ & $153,692.004$ \\
$\mathbf{I}(\mathbf{\%})$ & $\mathbf{8 8 . 3 6 6}$ & $\mathbf{5 5 . 2 7 1}$ & $\mathbf{8 8 . 8 5 5}$ \\
\hline
\end{tabular}

Table 4 Steady states defined by centralized optimization for the control system of Williams-Otto plant.

\begin{tabular}{llll}
\hline & $\boldsymbol{S S}_{\mathbf{1}}$ & $\boldsymbol{S S}_{\mathbf{2}}$ & $\boldsymbol{S S}_{\mathbf{3}}$ \\
\hline $\mathbf{F}_{\mathbf{A}}$ & $13,545.287$ & $13,550.959$ & $13,550.488$ \\
$\mathbf{F}_{\mathbf{B}}$ & $31,506.085$ & $31,503.076$ & $31,503.977$ \\
$\mathbf{F}_{\mathbf{R}}$ & $157,291.428$ & $157,297.190$ & $157,294.603$ \\
$\mathbf{F}_{\mathbf{R A}}$ & $18,166.939$ & $18,191.913$ & $18,192.398$ \\
$\mathbf{F}_{\mathbf{R B}}$ & $60,810.825$ & $60,800.771$ & $60,806.566$ \\
$\mathbf{F}_{\mathbf{R C}}$ & $3,331.909$ & $3,337.433$ & $3,337.809$ \\
$\mathbf{F}_{\mathbf{R E}}$ & $60,554.496$ & $60,543.003$ & $60,535.691$ \\
$\mathbf{F}_{\mathbf{R P}}$ & $10,818.450$ & $10,817.300$ & $10,816.569$ \\
$\mathbf{F}_{\mathbf{G}}$ & $3,608.809$ & $3,606.769$ & $3,605.569$ \\
$\mathbf{F}_{\mathbf{D}}$ & $36,679.563$ & $36,684.265$ & $36,685.896$ \\
$\mathbf{T}_{\mathbf{R}}$ & 655.893 & 655.849 & 655.840 \\
$\mathbf{I}(\mathbf{\%})$ & $\mathbf{8 8 . 3 3 3}$ & $\mathbf{5 5 . 1 7 0}$ & $\mathbf{8 8 . 8 3 5}$ \\
\hline
\end{tabular}

optimization, later moving on to scenario 2 and returning to scenario 1 . The value used as the initial estimate for the optimization is always the value of variables in the instant prior to the change.

Centralized optimization is also used to establish the optimum operating points to be compared with decentralized coordinated optimization results. Table 4 shows the values obtained with centralized optimization.

The yield obtained during the time of simulation (50 h), using decentralized coordinated optimization with the Direct Method, is 232.5\%, a slightly higher profit. This increase, nearly $0.1 \%$ at this time, represents an estimated rise of $1.4 \%$ monthly.
A decentralized optimization using the Direct Method ensures optimum operating points much closer, even for different scenarios when compared to centralized optimization. This makes unnecessary to take sudden changes in system to maintain the process in the most profitable point in the established conditions.

The control system proposed uses decentralized coordinated optimization to define steady state points. Figs. 6 and 7 show the results of control systems actions.

Once established an optimal point for the stationary state, it is noted that a relatively simple controller as the PI can keep the system in that point. 


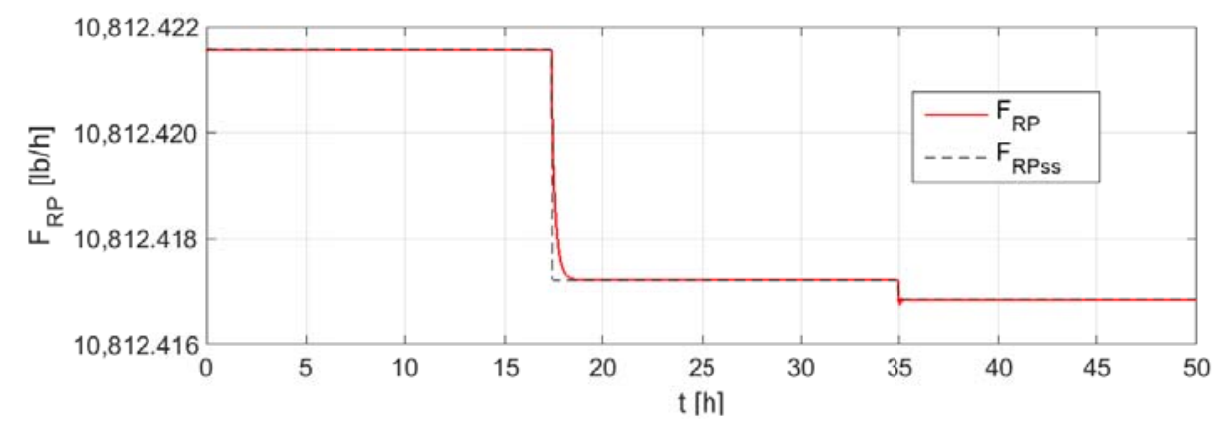

Fig. 6 Dynamic behavior of $F_{P R}$ to the control system proposed in Williams-Otto plant using the Direct Method.

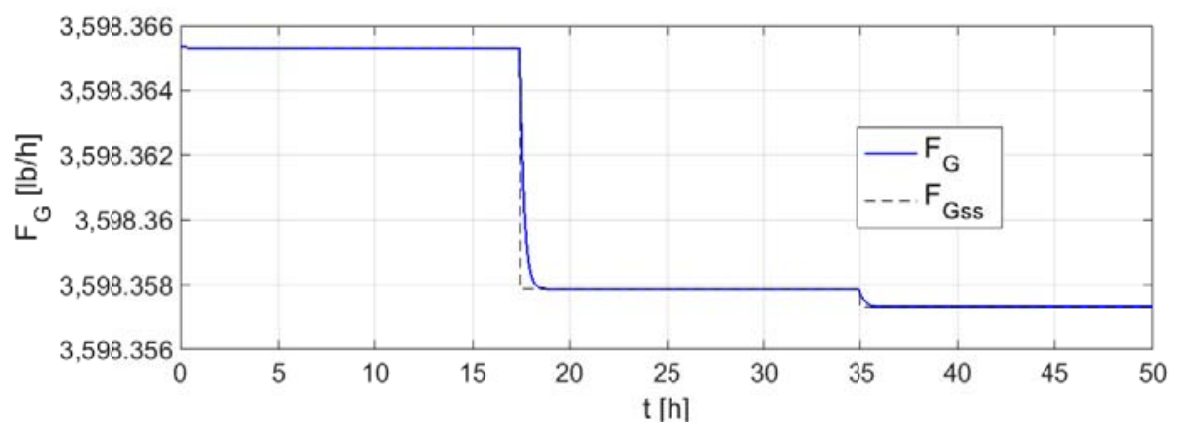

Fig. 7 Dynamic behavior of $F_{G}$ to the control system proposed in Williams-Otto plant using the Direct Method.
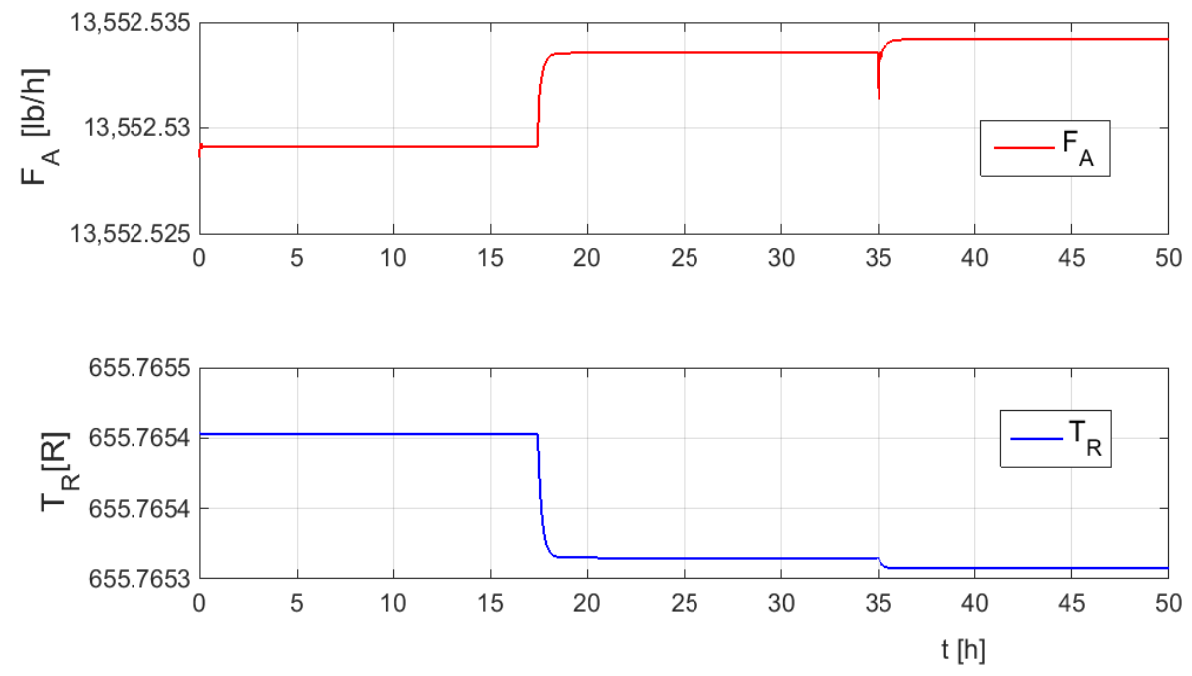

Fig. 8 Manipulation results of variables $F_{A}$ and $T_{R}$ from the control system to Williams-Otto plant using the Direct Method.

Dynamic behavior resultant is precise, without oscillations and performs the benchmark changes smoothly and accurately.

Fig. 8 presents the behavior of manipulated variables $F_{A}$ and $T_{R}$ from PI controllers.

Extreme control actions are not necessary in the manipulated variables so that they maintain the system at the desired point.
Fig. 9 shows $F_{B}$ and $F_{D}$ behaviors from open loop control decisions.

The proximity of the steady states defined by decentralized optimization makes small adjustments result in efficiency and accuracy of the control system to keep the system at the exact point to reach the established goal.

A same scenario was simulated with optimization 

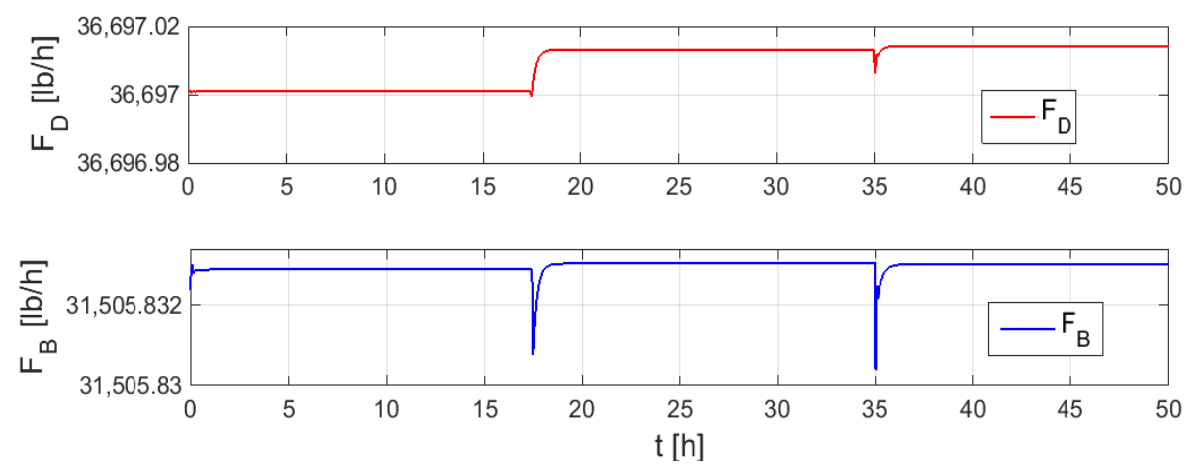

Fig. 9 Manipulation of variables $F_{B}$ and $F_{D}$ from the control system to Williams-Otto plant using the Direct Method.

Table 5 Steady states defined by decentralized coordinated optimization with the IBM for the control system of Williams-Otto plant.

\begin{tabular}{|c|c|c|c|c|c|c|}
\hline & \multicolumn{2}{|l|}{$\overline{S S_{1}}$} & \multicolumn{2}{|l|}{$S S_{2}$} & \multicolumn{2}{|l|}{$S S_{3}$} \\
\hline & $\lambda_{0}=\left[8.10^{-4}\right.$ & 8. $\left.10^{-6}\right]^{T}$ & $\lambda_{0}=\left[3.10^{-4}\right.$ & 1. $\left.10^{-6}\right]^{T}$ & $\lambda_{0}=\left[4,5 \cdot 10^{-5}\right.$ & $\left.1.10^{-6}\right]^{T}$ \\
\hline$\overline{\mathbf{F}_{\mathrm{A}}}$ & $13,100.087$ & & $13,227.510$ & & $13,025.832$ & \\
\hline $\mathbf{F}_{\mathbf{B}}$ & $32,025.464$ & & $32,269.405$ & & $32,333.472$ & \\
\hline $\mathbf{F}_{\mathbf{R}}$ & $159,591.265$ & & $161,546.898$ & & $160,814.456$ & \\
\hline $\mathbf{F}_{\mathbf{R A}}$ & $17,815.840$ & & $18,984.901$ & & $18,076.804$ & \\
\hline $\mathbf{F}_{\mathrm{RB}}$ & $65,337.550$ & & $67,327.467$ & & $67,398.550$ & \\
\hline $\mathbf{F}_{\mathbf{R C}}$ & 3,359.858 & & $3,674.172$ & & $3,466.184$ & \\
\hline $\mathbf{F}_{\mathrm{RE}}$ & $59,239.852$ & & $58,117.319$ & & $58,343.218$ & \\
\hline $\mathbf{F}_{\mathrm{RP}}$ & $10,686.985$ & & $10,574.732$ & & $10,597.322$ & \\
\hline $\mathbf{F}_{\mathbf{G}}$ & $3,151.179$ & & 2,868.308 & & 2,932.379 & \\
\hline $\mathbf{F}_{\mathbf{D}}$ & $36,697.002$ & & $36,697.002$ & & $36,685.896$ & \\
\hline $\mathbf{T}_{\mathbf{R}}$ & 653.691 & & 650.533 & & 651.999 & \\
\hline $\mathbf{R}$ & $115,394.058$ & & $114,960.399$ & & $115,672.646$ & \\
\hline Fc & $153,692.001$ & & $153,692.001$ & & $153,692.001$ & \\
\hline I(\%) & 89.141 & & 58.892 & & 85.363 & \\
\hline
\end{tabular}

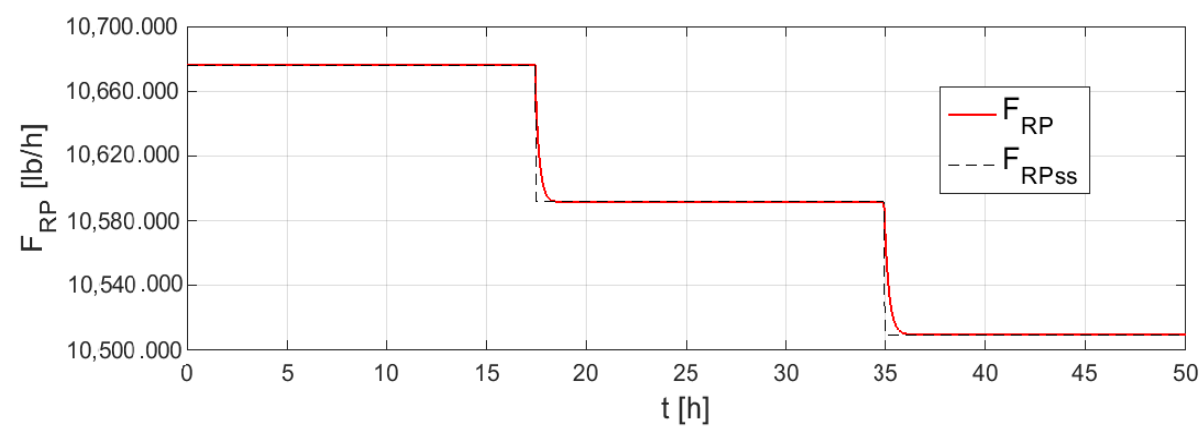

Fig. 10 Dynamic behavior of $F_{P R}$ to the control system proposed in Williams-Otto plant using the IBM.

layer utilizing decentralized coordinated optimization with the IBM. Table 5 presents optimal results found with this methodology.

Using the IBM in coordination step attains a yield $I=233.40 \%$ that represents a monthly increase, in average, of $6.6 \%$. Although there is no established theory on choosing the initial values for $\lambda$ yet, the results for the method are promising.

The control system designed to the system utilizes steady states defined by decentralized coordinated optimization to keep the system in optimal operational conditions. Figs. 10 and 11 show the behavior resulting of control actions to $F_{P R}$ and $F_{G}$.

Control system easily reaches the steady states 
determined by the IBM while maintaining the same tuning parameters throughout the simulation time.

The designed control systems do not require constant adjustments in tuning since they work with near reference points to each other, making small adjustments just to maintain the system in the optimal (economically) point.

Fig. 12 presents manipulated variables $F_{A}$ and $T_{R}$ from PI controllers and Fig. 13 shows $F_{B}$ and $F_{D}$ behavior from open loop control decisions.

It can be noticed that even in times of change of operating points, the control stays robust, with smooth actions and without overshoot, not requiring too much of the manipulated variables to keep its objective.

The manipulation of variables to achieve the control objectives is even smoother when using the steady state set by the IBM. The controller has a greater freedom of handling when the reference values are not as close as in the Direct Method.

Although it is an advantage to get near steady states of each other, this limits the manipulation of system entries thus justifying the need to tune the controller at every change of optimal point.

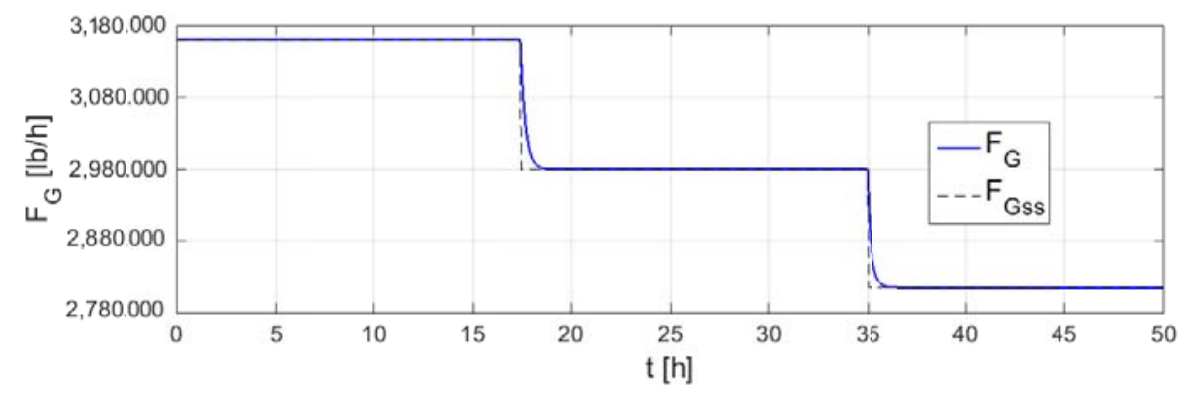

Fig. 11 Dynamic behavior of $F_{G}$ to the control system proposed in Williams-Otto plant using the IBM.
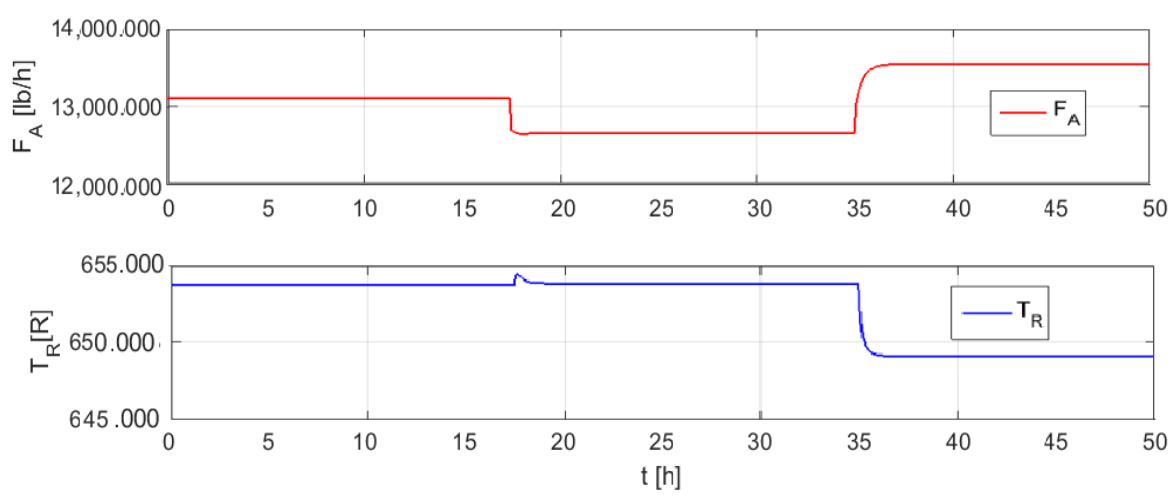

Fig. 12 Manipulation results of variables $F_{A}$ and $T_{R}$ from the control system to Williams-Otto plant using the IBM.
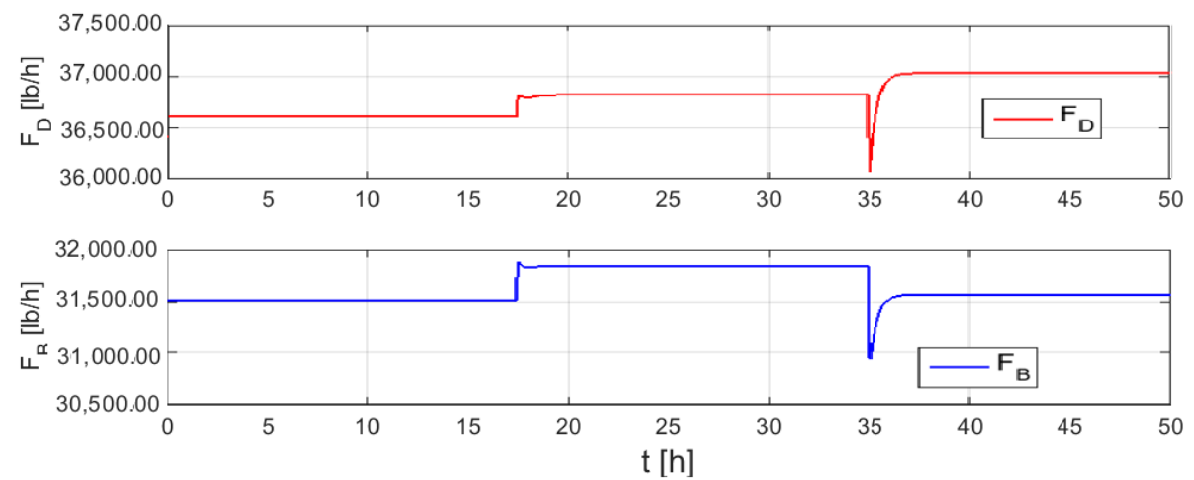

Fig. 13 Manipulation of variables $F_{B}$ and $F_{D}$ from the control system to Williams-Otto plant using the IBM. 


\section{Conclusions}

From the results found for the coordinated decentralized optimization, it can be considered that the optimal point found is very close to the optimal centralized optimization, presenting minor adjustments in variables so that such point meets not only the requirements of local problems, but also of the entire plant.

The decentralized optimization is a viable and efficient alternative to the centralized optimization, being an option for large-scale systems with lower computational requirements for solving less complex problems than the optimization problem of a global plant of a process.

It is noteworthy that although the increase provided in the objective function of the system may not appear to be numerically significant, when it is related to the profit of a company, estimated values in millions, an increase can make it a still more attractive process economically.

Utilizing the decentralized optimization as an auxiliary tool in the control design of entire plants, the use of intersecting variables of process subsystems helps in minimizing the effects of the manipulation in the input variables in the other subsystems, as the optimal point was defined by considering many interactions between them.

The planning production association with the control of a process allows the control system to take smoother and more accurate actions in order to maintain the system in optimum, as it presents significant economic benefits for the operation of complex chemical plants.

\section{References}

[1] Hori, E. S. 2005. “A Contribuition to Plantwide Control: Indirect Control and Coordination of Decentralized Controlers.” Ph.D. thesis, UFSCar (Universidade Federal de São Carlos).

[2] Andrade, G. V. N. 2008. "Design of Control Structure to a Planto of Etanol Production.” Master thesis, Universidade Federal do Rio de Janeiro (COPPE/ UFRJ).
[3] Morud, J., and Skogestad, S. 1996. "Dynamic Behavior of Integrated Plants.” Journal of Process Control 6 (2/3): 145-56.

[4] Luyben, W. L. 1993. "Dynamics and Control of Recycle Systems. 1. Simple Open-Loop and Closed-Loop Systems." Industrial and Engineering Chemistry Research 32: 466-75.

[5] Bansal, V., Ross, R., Perkins, J. D., and Pistikopoulos, E. N. 2000. "The Interactions of Design and Control: Double-Effect Distillation.” Journal of Process Control 10: 219-27.

[6] Skogestad, S. 2004. "Control Structure Design for Complete Chemical Plants.” Computers and Chemical Engineering 28: 219-34.

[7] Araujo, A. C. B. 2007. "Studies on Plantwide Control." Ph.D. thesis, Norwegian University of Science and Technology.

[8] Stephanopoulos, G., and Ng, C. 2001. "Perspectives on the Synthesis of Plantwide Control Structures.” Journal of Process Control 10: 97-111.

[9] Larsson, T., and Skogestad, S. 2000. "Plantwide ControlA Review and a New Design Procedure.” Modeling, Identification and Control 21 (4): 209-40.

[10] Buckley, P. S. 1964. Techniques of Process Control. New York: Wiley.

[11] Luyben, W. L., Tyréus, B. D., and Luyben, M. L. 1998. Plantwide Control Process. New York: Mc-Graw-Hill.

[12] Narraw, L. T., and Perkins, J. D. 1993. "Selection of Process Control Structure Based on Linear Dynamic Economics.” Industrial and Engineering Chemistry Research 32 (1): 2681-92.

[13] Degliuomini, L. N., Zumoffen, D., and Basualdo, M. 2012. "Plantwide Control Design for Fuel Processor System with PEMFC.” International Journal of Hydrogen Energy 37 (19): 14801-11.

[14] Kwong, W. H. 1992. "Optimization of Industrial Complex Plants.” Ph.D. thesis, Universidade de São Paulo.

[15] Badeal, M., and Harjunkoski, I. 2014. "Integrated Production Scheduling and Process Control: A Systematic Review.” Computers and Chemical Engineering 71: 377-90.

[16] Williams, T. J., and Otto, R. E. 1960. “A Generalized Chemical Processing Model for Investigation of Computer Control.” AIEE Trans 79: 458-68.

[17] Jung, B. S., Miroshi, W., and Ray, W. H. 1971. "Large Scale Process Optimization Techniques Applied to Chemical and Petroleum Process.” The Canadian Journal of Chemical Engineering 49 (12): 844-52.

[18] Biegler, L. T., and Zavala, V. M. 2009. "Large-scale Nonlinear Programming Using IPOPT: An Integrating Framework for Enterprise-wide Dynamic Optimization.” 


\section{Williams-Otto Plant Control Based on Production Planning Associated to Coordinated Decentralized Optimization and Plantwide Control Techniques}

Computers and Chemical Engineering 33: 575-82.

[19] Leineweber, D. B., Bauer, I., Bock, H. G., and Schölder, J. P. 2003. "An Efficient Multiple Shooting Based Reduced SQP Strategy for Large-Scale Dynamic optimIzation. Part 1: Theoretical Aspects.” Computers and Chemical Engineering 27: 157-66.

[20] Zavala, V. M., Laird, C. D., and Biegler, L. T. 2008. "Interior-point Decomposition Approaches for Parallel Solution of Large-Scale Nonlinear Parameter Estimation Problems.” Chemical Engineering Science 63: 4834-43.

[21] Harjunkoski, I., and Grossmann, I. E. 2001. "A Decomposition Approach for the Scheduling of a Steel Plant Production.” Computers and Chemical Engineering 25: 1647-60.

[22] Basset, M. H., Pekny, J. F., and Reklaitis, G. V. 1996. "Decomposition Techniques for the Solution of Large-Scale Scheduling Problems.” AIChE Journal 42 (12): 3373-87.

[23] Shh, N., Saharidis, G. K. D., Jia, Z., and Ierapetritou, M. G. 2009. "Centralized-Decentralized Optimization for Refinery Scheduling." Computers and Chemical Engineering 33: 2091-105.
[24] Gnoni, M. G., Iavangnilio, R., Mossa, G., Mummolo, G., and Di Leva, A. 2003. "Production Planning of a Multi-site Manufacturing System by Hybrid Modelling: A Case Study from the Automotive Industry.” International Journal of Production Economics 85: 251-62.

[25] Inalhan, G., Stipanovic, D. M., and Tomlin, C. J. 2002. "Decentralized Optimization, with Application to Multiple Aircraft Coordination.” In Proceedings of the 41st IEEE Conference on Decision and Control, 1147-55.

[26] Findeisen, W., Bailey, F. N., Brdýs, M., Malinowski, K., Tatjewski, P., and Wozniak, A. 1980. "Control and Coordination in Hierarchical Systems.” International Series on Applied Systems Analysis. New York: John Wiley and Sons.

[27] Bakallis, P. S., and Ellis, J. E. 1989. "Simulation of Hierarchical Control Algorithms Using a Steady-State Mathematical Model of a Vaporizer Plant.” Appl. Math. Modelling 13: 413-9.

[28] Flórez, J. Z., Martinez, J., Besançon, G., and Faille, D. 2013. "Decentralized-Coordinated Model Predictive Control for a Hydro-Power Valley.” Mathematics and Computers in Simulation 91: 108-18. 